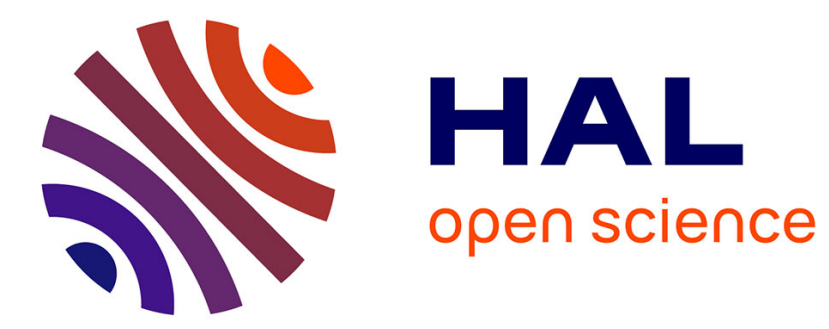

\title{
From Research on the virtual reality installation
}

François de Sorbier, Patrice Bouvier, Adrien Herubel, Venceslas Biri

\section{To cite this version:}

François de Sorbier, Patrice Bouvier, Adrien Herubel, Venceslas Biri. From Research on the virtual reality installation. Human-Computer Systems Interaction: Background and Application, 2009, 60 (1), pp.335-345. hal-00681737

\section{HAL Id: hal-00681737 https://hal.science/hal-00681737}

Submitted on 22 Mar 2012

HAL is a multi-disciplinary open access archive for the deposit and dissemination of scientific research documents, whether they are published or not. The documents may come from teaching and research institutions in France or abroad, or from public or private research centers.
L'archive ouverte pluridisciplinaire HAL, est destinée au dépôt et à la diffusion de documents scientifiques de niveau recherche, publiés ou non, émanant des établissements d'enseignement et de recherche français ou étrangers, des laboratoires publics ou privés. 


\title{
From Research on the virtual reality installation
}

\author{
F. de Sorbier de Pougnadoresse, P. Bouvier, A. Herubel and V. Biri
}

Université Paris-Est, Laboratoire d'Informatique de l'Institut Gaspard Monge (LABINFO-IGM), UMR CNRS 8049, France

\{fdesorbi, bouvier, herubel, biri\}@univ-mlv.fr

Summary. This article presents a new virtual reality installation inspired by the CAVE system but non expensive (7000€) and transportable. Moreover, we especially focus on the feeling of presence. This means user should be able to feel his self-presence in the virtual environment and the presence of the virtual objects and entities. Keeping in mind to increase the feeling of presence, we finally propose a dedicated game based on an immersive environment, consistent interactions and emotions.

\section{Introduction}

Virtual reality (VR) aims at plunging one or more users at the heart of an artificial environment where they are able to feel and interact in real time thanks to sensori-motor interfaces. The key-point of a VR experiment is that users respond through real actions and emotions to stimuli which materialize the virtual environment (VE) and the virtual events.

VR is a leading area which is mainly exploited in high-end fields like simulators, scientific research, army and industry. Reasons why VR is not more generalized are mainly the cost of the system, the large room needed and the skills required. However, there is a growing demand for the use of VR in fields like games, artistic installations and education. That's why we design a non-expensive (7000€) CAVE-like system [1] which is also easily transportable in order to be deployed in school or during show.

Our system use four screens of size $3 \mathrm{~m} \times 3 \mathrm{~m}$, a 3D sound rendering with headphones and various interaction tools like the Nintendo Wiimote or a $3 \mathrm{mx} 3 \mathrm{~m}$ homemade sensitive carpet. After an overview of the concepts related to presence, we will describe our VR installation. Finally, we will outline the results of the evaluation of the system. 


\section{Virtual reality and presence}

\subsection{The role of presence}

VR experience makers often intend to create the conditions required to make users feel presence. This feeling of presence could be seen shortly as a strong feeling to exist in the VE. This means, user's being in the VE has to be the most natural as possible in the sense without the awareness of the virtuality of the situation [2]. Even if it has not been yet strongly established that presence improve the efficiency of the experiment $[3,4]$, presence remains an important aims to tend toward in that it motivates the researches on several levels: new interaction devices, new algorithms etc.

The feeling of presence can be seen from three angles [5]. Firstly the spatial presence or being there [6] which is to feel like at a different place. Self-presence, here it's not being there but just being, that is projection of user's ego in the role he is supposed to incarnate in the VR application. Social presence is the being with, it relates to the presence of other intelligences.

One understands that to tend toward such unstable psychological state, it is crucial that users accept to get caught up in the experiment. Then, our work is to provide an experiment sufficiently credible -but not necessarily realistic- in order to delude user's sense and critical thinking. For this purpose we identified five interrelated technical and psychological pillars on which we can lean on: human cognition knowledge as a foundation, immersion, interaction, consistency of the sensori-motor loop and emotions. These pillars, which are inclined to be sufficiently generic to fit with any kind of application field, have to be seen as guidelines for VR designers. We point the fact that there is no hierarchy between the pillars, some are technical, others are more human side. Sometimes they work on their own, sometimes they work in conjunction, the aim still remains to arouse presence in a synergistic manner.

\subsection{The pillars of presence}

As we already said human must be at the heart of a VR system that's why it is central to understand how we perceive our environment and other humans, how we construct a mental representation of the environment etc. Moreover this may also permit to understand "the mental and neural processes that may underlie presence" [2]. That's why we consider human cognition knowledge as a root for other pillars. We could lean on the headways in neuropsychology, social cognition, psychology of emotions etc. This knowledge may guide the VR system design (how to delude user's senses?) but also the experiment itself (scenario, atmosphere). 
The second pillar is immersion. It is achieved through the stimulation of user's senses in order to generate sensations which enable, sometimes thanks to an illusion, the perception of the virtual environment. From this perception will ensue a proper comprehension of the virtual environment and consequently its appropriation. At the stage of immersion there is absolutely no reference to presence or being there. The immersion is measurable: does the system provide a stereoscopic display? a 3D sound spatialization? use a sensory substitution? show aesthetic high-defined textures etc. It is obvious that immersion will be more complete [7] if several senses are stimulated in coherence. Last point, most of the time the virtual environment is rendered as it would be if no user were there. We forget that, if someone is plunging in a virtual environment, by his simple inactive presence he has an effect on this environment. So we claim that immersion must be bi-directional, this will improve the credibility of the environment.

The third pillar, interaction has to fulfill two tasks : to acquire information (for example via a tactile system) and to communicate information (for example to point out or manipulate an object, to modify the environment, to navigate etc. ). Moreover, by inducing that user and virtual entities exist in the VE because they interact, interactions possibilities will help to improve the feeling of presence. If we refer to the concept of "perceptual illusion of non mediation" from [8] we understand that interaction devices must be as transparent and natural as possible until being forgotten. Moreover, direct body interactions could improve presence because these latter enable to match virtual actions with real or at least expected proprioceptive and kinesthetic feedback. It could be the case if a virtual movement corresponds to a real movement like walking for example. Finally, until now we only emphasized communication between user and the environment but of course that can include a communication with autonomous agents or other human.

The fourth pillar consists in maintaining an action-perception or sensori-motor loop consistent. It will be necessary to respect two main points. Firstly, we will have to take care not to break the causality link between user's actions and the system's feedback. That means we have to implement real-time algorithms and provide high frequency displays. Secondly, we must maintain the time and place consistency between various sensory stimulations associated with the same event or virtual object. This fourth pillar constitutes a link between the last two ones but can also include some works related to synesthesia, cross-modal illusion $[9,10]$, multi-modality [11].

Even by providing some high quality immersion and interaction which respect the consistency of the action-perception loop, it will probably remain a distance between users and the role they are suppose to incarnate in the experiment. This distance which links user to reality may have several causes: the system's shortcomings, the real world distractions [12] or just because the experiment is not motivating. We argue that technology can not, on its own, create the feeling of presence $[13,14]$. Here is the role of the emotion pillar. By introducing emotion in the experiment we want to cancel this distance and encourage users to forget these disturbances and accept to get caught up and stay focused on the experiment. Fi- 
nally, we think that emotions and presence self support themselves in a virtuous circle: firstly, emotion enables to reach presence more easily and then presence permits to feel more intensely emotions [14].

In section 3 we will explain how we put some of these ideas in concrete form through different choices.

\subsection{Virtual reality installations}

Head mounted displays [15] (HMD) are often associated with the concept of virtual reality because they seem to be well-suited for immersive purposes. That kind of device which is quite intrusive because of weight, is composed of two screens allowing stereoscopic display. A strength and a weakness of a $H M D$ is that users are roughly and completely cut off from the real environment. Therefore, the loose of fixed points contributes to faze user who will be unable to keep a motionless position. Moreover, its cost will restrain its use to single user's applications. Finally, it is necessary to operate a tracking of user's position to maintain the consistency between the virtual content and the orientation of the viewpoint. For these reasons, we prefer to concentrate on screen-based installations.

The $C A V E$-system [1] relies on large screens to increase the feeling of presence, placing user at the center of a cube. Each face is a screen on which stereoscopic images are back-projected. That way, user is visually plunged into the virtual world (and drawbacks of $H M D$ 's are almost removed). However, like starcave [16], SAS, blue-c [17] or Allosphere [18], a CAVE-like system requires large space to ensure a correct display area. Furthermore, such an installation is hardly transportable due to its size and meticulous settings that are required. Finally, this system may be expensive because of devices used, maintenance cost and framework needed.

These limits have led to propose some alternatives. The area taken up by the VR installation can be reduced by replacing the projective devices with welladapted ones. For example, The Varrier [19] suggests to use walls of autostereoscopic screens instead of the back-projected screens but this solution is very expensive. Trying to ease the displacement of an installation, MiniVR [20] and $C y$ berdome [21] suggest solutions based on a small screen or a curved screen.

To cut prices, [22,23] and HIVE [24] have followed a self-made approach with consumer grade components. In addition, these installations are easily transportable because they are light and simple to assemble. Concretely, these VR installations bring flexibility and control of the system.

\section{About our virtual reality installation}




\subsection{Outlook}

Here are the constraints we identified in order to create a VR system less expensive and easily transportable. Software has to be home-made or free opensource, bulk of our system has to be designed with consumer grade components and if no low-cost solution exists for a device we create it. Moreover, the framework has to be as light as possible, easy to assemble, a medium room like a class room must contain it and the whole system has to be transportable in a commercial vehicle.

Despite these constraints we still want to provide an experiment which enables to feel presence. So, we will have to take care of several points according to each pillars. For immersion : the quality of the video-projector (luminosity, resolution, frequency, size of the projection plane), multisensorial rendering (sound, tactile). For interactions, we provide natural devices which are not too much intrusive but offer a good precision (Wiimote, sensitive carpet for the management of user's moves). For the consistency of the sensorimotor loop : low latency system based on a client-server architecture. And finally, to find some subterfuges in the application to make user forget the shortcomings of the installation.

\subsection{Description of the installation}

\section{The screens}

To visually immerse the user we choose to use a $C A V E$-like display system but using only the four vertical faces of the cube. The upper face is not used because the installation is designed to be used in rooms with classical height (roughly $3.50 \mathrm{~m}$ ). Each screen is hand-made using tracing paper which is resistant and allows back-projection. A video-projector is placed behind each screen and is calibrated as for the image perfectly matches its surface. This layout prevents user to cast his shadow when working close to the screen.

Currently, the visual installation is monoscopic but can easily evolved to stereoscopic display. The installation can be upgraded with twice more video-projectors and special screens which support a polarized projection system [25]. However the number of required devices does not allow us to experiment stereoscopic projection yet. Active devices could also be used but need to be synchronized and are still expensive. 

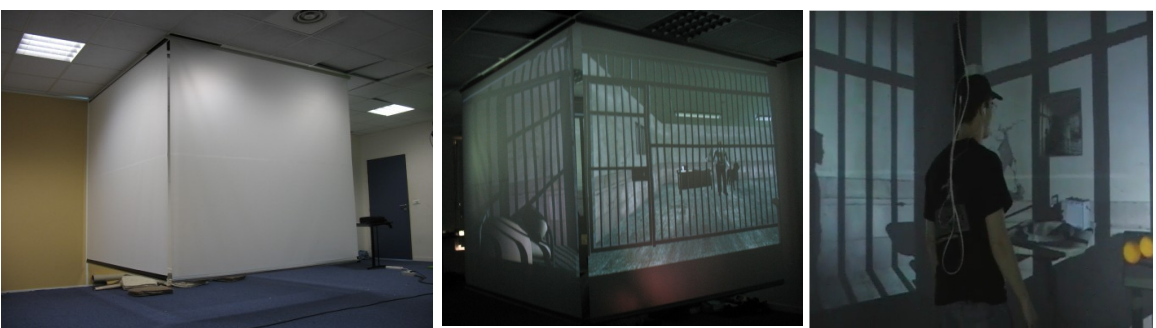

Fig. 1. Different views of our virtual reality installation

\section{Sound spatialization}

To render the virtual acoustic environment two kinds of devices can be used: headphones or loudspeakers each having their advantages and shortcomings. Headphones are generally considered as more intrusive than loudspeakers what hinders the immersion. But headphones have the significant advantage of isolating the listener from external sounds (spectators, other installations etc.). As our installation is transportable, it can be installed in halls where the acoustic cannot be controlled and thus phenomenon of reverberation could appear and perturb the listener's perception. That is why we decide to use headphones as auditory display and a simple implementation of the HRTF associated to a spherical head model [26].

\section{Interaction devices}

One way to interact with the virtual environment is to capture user's position and head's orientation. Keeping in mind the cost constraint, two devices have been created.

The first device is a multi-zonal sensitive carpet used to capture user's position. User's weight enables the contact of two aluminum sheets isolated by perforated foam (Fig. 2 right). The floor area of the carpet is $9 \mathrm{~m}^{2}$ and contains 64 square zones meaning that the precision of the device is $14 \mathrm{~cm}^{2}$. Data transmission between the carpet and the server is provided by a MIDI interface. The multi-zonal sensitive carpet transmits the approximate user's feet position and some actions can be detected like user's jump or when user is hitting the ground with one of his feet. User's average posture can be deduced when these latter information are coupled with the compass data. 
The second device is indeed an electronic compass that gives user's head orientation. The lightness of the sensor allows placing it on a cap worn by user so its impact on the feeling of presence is small. The device is depicted on Fig. 2.
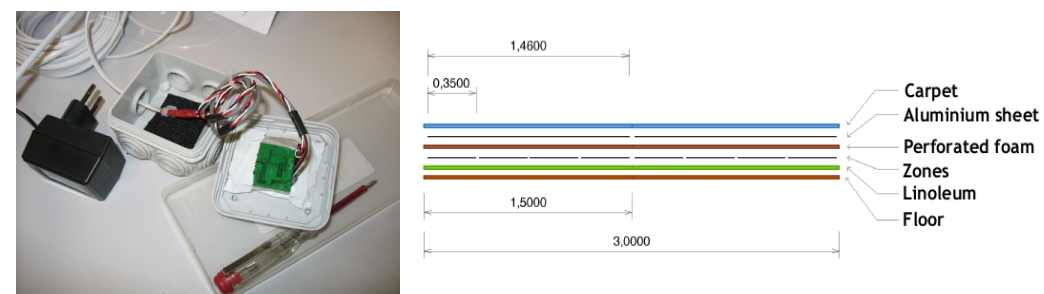

Fig. 2. The electronic compass (left) and a cross section of the sensitive carpet (right)

A Nintendo Wiimote is used to let the user directly interacts with the content of the virtual environment. The benefits are its low price and the capacities of that device to increase immersion. Moreover, some actions can be triggered according to user's position captured by the sensitive carpet.

\section{Software architecture}

Achieving real-time is a key point for an immersive installation and software architecture is the corner stone of this accomplishment. Such installation runs numerous processing threads sometimes very complex like sound processing, captor acquisitions and 3D rendering on multiple displays. Those processes are mostly independent from each other except for synchronization data. Such characteristics call for a distributed architecture over network. Assigning processes between several machines reduces per machine computational overhead and assures that every process gets the needed level of preemption. However this solution does have structural problems, so we stated that different processes need synchronization data. As an example, sound processing needs to be synchronized with user's position. Network accesses, despite being nearly as fast as hard disk accesses, are significantly slower than RAM and carrying data between each machine can be a huge bottleneck. The lack of hard real-time virtual reality platforms is another problem. Hard real-time ensures that two machines A and B receiving a signal from a machine $C$ will act exactly the same at the same time. To address this problem A and B should share a clock signal to synchronize their reactions to an event. 
Our implementation uses a client/server architecture pattern with a double logical network topology. Our server hosts several processes and handles the central control of the installation. An operator can diagnose problems and possibly stop or restart the experiment. In order to avoid multiple synchronizations between captors, we centralized their acquisitions on the server.

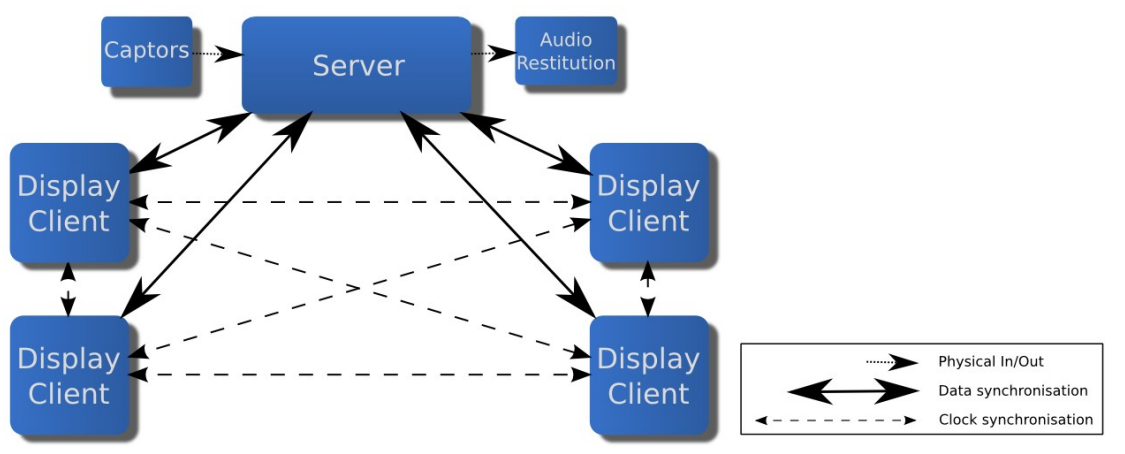

Fig. 3. Software architecture

The server hosts the sound processing and runs threads as well. Sound data should not be carried through the network since it may significantly decrease network performances. Then, only sound controls are carried over the network through the sound thread, which may process and play a locally stored sound as a consequence. Our server is a completely homemade $C++$ program using the $Q t$ library for inputs/outputs and the GUI. In our installation, the most resource intensive process is the multiple screen $3 \mathrm{D}$ rendering. Each $3 \mathrm{D}$ rendering process is a client to our server and is hosted on a separate machine. In our installation a display client is a Ogre $3 D$ application, but most of 3D engines such as OpenSceneGraph could be used as a display client. Each client is able to render on one screen and can be informed of captors data. It can order the server to play or stop a particular sound. Synchronizing this events with the server is achieved using a star logical network. Signals from the server are broadcasted to each client while clients orders directly reach the server. We stated that when receiving the same signal, clients can act slightly differently due to the lack of hard real-time. We resolve this problem with a full mesh logical network topology which connects every client. Each client broadcast a clock signal on the network to other clients. The clock is a signal count. If a client detects that another one has received more signals it will wait to receive the same signal before triggering more events. Else, if a client detects that the others didn't receive a signal, it will wait for them. This simple mechanism avoids desynchronization between clients. 


\subsection{Importance of the content}

As described previously, one of the pillars concerns emotions. Most of virtual reality installations include computing units and others devices which lead to create visual and audio discomforts. More the user feels involved in the world presented to him more he forgets these imperfections. Generating emotions helps captivating user to focus his attention on the virtual environment rather than the material. To that purpose, we create a scenario taking advantage of the emotion's pillar to involve users.

Several video games studies $[27,28]$ have shown the importance of integrating emotions in content to increase presence feeling. For example, fear and anxiety are widely used for stimulating user's emotions in video games implementing dark atmosphere or dangerous place. Considering the scenario, it should be an help for the generation of emotions [29].

For these reasons, our VR installation has been developed considering using emotions within a scenario. We created a story for first person game taking place in a prison. The user is playing the role of a prisoner who is trying to escape with a fellow prisoner (NPC) who gives some advice. That scenario has several advantages. First it allows user to evolve in the installation as he is moving in the virtual environment (for example the floor area fit with the jail area). Secondly, the atmosphere of prison is associated with strong feelings of stress and fear. These two latter points contribute to induce the feeling of presence, by generating emotions that involve user in the virtual world, and by increasing immersion thanks to similarities between real and virtual spaces.

Bi-directional aspect of immersion is also increased by displaying user's shadow and his image on reflective surfaces like mirrors. This point is important for self-presence as user has to feel existing in the virtual world. For that purpose, we use a technique named visual hulls [30] which allows to reconstruct the geometry of an object according to a set of images captured from calibrated cameras. For each image, the silhouette of the object is computed and unprojected in the form of a cone centered on the related camera. The intersection of the cones produces an envelope approximating the body of the object. In the same way, we use visual hulls to create an avatar similar to the user which will be used to compute shadow or reflections in a mirror as in figure 4. 


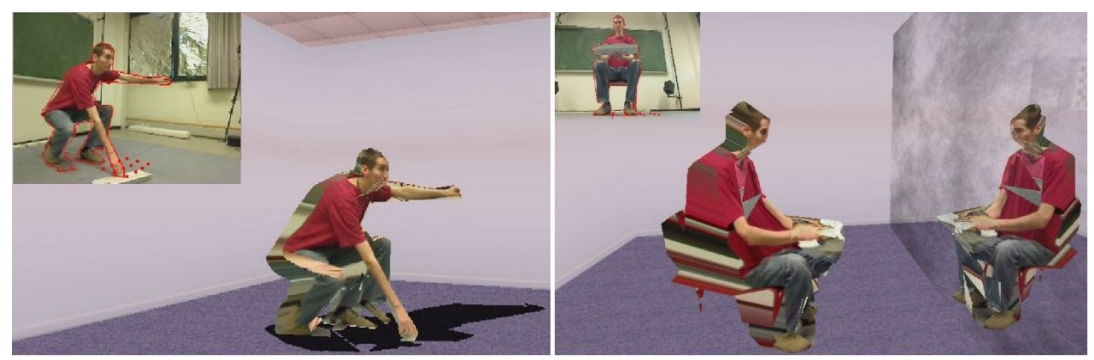

Fig. 4. Using a visual hulls of the user increase self presence

\subsection{Financial details}

As previously explained, the main disadvantage of most VR installations is their cost. Especially, CAVE-like systems are expensive (from 23,000€ to $300,000 €)$. These reasons led us to propose a low-cost installation made with consumer grade components. The manufacturing cost of our installation is around $7,500 €$ divided as follows :

- Display (4 screens and 4 video-projectors) : 3,800€

- Computer hardware (5 PCs with Linux and network) : 3,300€

- Small equipments (carpet, electronic compass and headphones) : $420 €$

\section{Evaluation}

We have initiated an evaluation procedure of the efficiency of the installation concerning the feeling of presence and to validate several of our choices detailed in section 3. Our study had 30 participants who are all graduates students in computer sciences. Most of them regularly play video games. For this evaluation we use post-exposure questionnaire using a seven point Likert item. We firstly evaluate the perceived feeling of presence through its different angles (cf. section 2.1). Question 1 about spatial presence : "During the game, how much did you feel like you were really there in the virtual environment ?" (1=there, $7=$ not there at all). Question 2 about self-presence : "During the game, how much did you feel you really be the prisoner ?" (1=was, 7=was not me at all). Question 3 about environment presence : "During the game, how much did you feel that the virtual environment was a real place ?" (1=real, 7=not real at all). Question 4 about social presence : "During the game, how much did you feel that other prisoners were existing person ?" (1=existing, $7=$ not existing at all). Here are the results (average of the 
quote) for these 4 questions: Q1: 3.5, Q2: 3.9, Q3: 4.1, Q4: 5.1. The results were quite conformed to our expectation, but we estimate them too unfinished to draw conclusions.

We also evaluate the relevance of our interaction tools choices. Question 5 : "Does the video game you just played provide high quality play control ?"(1=high quality, 7=low quality). We had a quite surprising bad result for this question which may show that people expect more from a VR game than an interaction tool like Wiimote.

In a future experiment we will evaluate the impact of each pillars on presence, independently and in conjunction. We will test if self-presence decrease without the virtual shadow or the reflect in the mirror. We will also do a comparative study between playing the game in front of a PC or in our installation.

\section{Conclusions}

We have presented a low-cost $(\sim 7000 €)$ and transportable version of a CAVElike virtual reality installation. We also take care to keep enough quality to arouse feeling of presence. We have described the different parts of the installation which are screens, sound, interaction devices and software architecture. We have also argued the importance of the content of the application to maintain the feeling of presence. Finally, we have briefly presented the results of our studies based on a questionnaire which were aimed at checking if user feel to be present in the virtual world.

These latter evaluations have pointed out the lack of efficiency concerning the pillar of interaction. So we are thinking to more natural solutions of interactions by using non-intrusive devices. One of our goals is to propose a direct interaction thanks to the tracking of user's hands. We would like to establish some eye contact during the experiment which will signify that you are existing for someone else and then will increase your feeling of self-and co-presence. We also would like to add several fans in the installation to simulate air displacement to increase immersion. Finally, we want evaluate the impact of our four screens display on the feeling of presence by comparing it with a standard PC display.

\section{References}

1. Cruz-Nera C, Sandin D J, Fanti T A, Kenyon R V and Hart J (1992). The CAVE: audio visual experience automatic virtual environment. In Communications of the ACM, 35, 6:64-72.

2. IJsselsteijn W A (2002). Elements of a multi-level theory of presence: Phenomenology, mental processing and neural correlates. In Proceedings of PRESENCE 2002:245-259.

3. Welch R B (1999). How Can We Determine if the Sense of Presence Affects Task Performance?, Presence, 8, 5:574-577.

4. Bormann K (2006). Subjective performance. Virtual Reality. 9, 4:226-233. 
5. Lee K M. (2004). Presence, explicated. Communication Theory, 14:27-50.

6. Minsky M (1980). Telepresence. Omni:45-51.

7. Stein B and Meredith M (1993). The Merging of the Senses. Cambridge, MA.

8. Lombard M and Ditton T (1997). At the heart of it all: The concept of presence. In journal of Computer Mediated Communication, 3(2).

9. IJsselsteijn W A, de Kort Y A and Haans A (2006). Is This My Hand I See Before Me? The Rubber Hand Illusion. In Reality, Virtual Reality, and Mixed Reality. Presence: Teleoper. Virtual Environ. 15, $4: 455-464$

10. Biocca F, Inque Y, Polinsky H, Lee A and Tang A (2002). Visual cues and virtual touch: Role of visual stimuli and intersensory integration in cross-modal haptic illusions and the sense of presence. Proceedings of Presence 2002.

11. Väljamäe A, Larsson P, Västfjäll D and Kleiner M (2005). Travelling without moving: Auditory scene cues for translational selfmotion, Proceedings of International Conference on $\mathrm{Au}$ ditory Display.

12. Wang Y, Otitoju K, Lu T, Kim S and Bowman D (2006). Evaluating the Effect of Real World Distraction on User Performance in Virtual Environments. In proceedings ACM Symp. Virtual Reality Software and Technology, ACM Press:19-26.

13. Banos R M, Botella C, Liano V, Guerrero B, Rey B and Alcaniz M (2004). Sense of presence in emotional virtual environments. Paper presented at Presence 2004: The 7th Annual International Workshop onPresence, Valencia.

14. Riva G, Mantovani F, Capideville C S, Preziosa A, Morganti F, Villani D, Gagioli A, Botella $\mathrm{C}$ and Alcaniz M (2007). Affective interactions using virtual reality: the link between presence and emotions. Cyberpsychology and Behavior, 10:45-56.

15. Sutherland I E (1968). A head-mounted three dimensional display. In Seminal Graphics: Poineering Efforts that Shaped the Field ACM, New York:295-302.

16. DeFanti T A, Dawe G, Sandin D J, Schulze J P, Otto P, Girado J, Kuester F, Smarr L and Rao R (2009). TheStarCAVE, a Third-Generation CAVE and Virtual Reality OptIPortal. To appear in Future Generation Computer Systems,25,2:169-178

17. Spagno C and Kunz A (2003). Construction of a Three-sided Immersive Telecollaboration System. In Proc. of IEEE VR 2003:22-26.

18. Hollerer T, Kuchera-Morin J and Amatriain X (2007). The Allosphere: A Large-Scale Immersive Surround-View Instrument. In Proceedings of the 2007 Emerging Display Technologies Workshop, San Diego, California.

19. Peterka T, Sandin D J, Ge J, Girado J, Kooima R, Leigh J, Johnson A, Thiebaux M and DeFanti T A (2006). Personal varrier: autostereoscopic virtual reality display for distributed scientific visualization. Future Gener. Comput. Syst. 22, 8:976-983.

20. Fairen M, Brunet P and Techmann T (2004). MiniVR: a portable virtual reality system, Computers \& Graphics, 28, 2:289-296.

21. Shibano N, Hareesh P, Hoshino H, Kawamura R, Yamamoto A, Kashiwagi M and Sawada K (2003). Cyberdome: Pc clustered hemi spherical immersive projection display. In Proc. of the 2003 International Conference on Artificial Reality and Telexistence:1-7.

22. Ohno R and Ryu J (2007). Development of a portable of a portable virtual reality system for disaster education. 8th eaea-Conference in Moskow.

23. Grimes H, McMenemy K R and Ferguson R S (2008). A transportable and easily configurable multi-projector display system for distributed virtual reality applications. In proceedings SPIE 6804:68040G-68040G-9.

24. Cliburn D C and Stormer K (2005). The HIVE: Hanover immersive virtual environment. J. Comput. Small Coll. 20, 4:6-12.

25. Bouvier P, Chaudeyrac P, Loyet R, Piranda B and de Sorbier F (2006). Immersive visual and audio world in 3D. In 9th International Conference on Computer Games:159-165.

26. Cheng C I and Wakefield G H (2001). Introduction to head related transfer functions (HRTFs):

Representation of hrtfs in time, frequency and space. In journal of the Audio Engineering Society, 49,4:231-249. 
27. Grimshaw M (2008). Sound and Immersion in the First-Person Shooter. In International Journal of Intelligent Games \& Simulation 5,1.

28. Schneider E F, Lang A, Shin M, Bradley S D (2004). Death with a story: How story impacts emotional, motivational, and physiological responses to first-person shooter video games. Human Communication Research, 30:361-375.

29. Aylett R and Louchart S (2003). Towards a narrative theory of virtual reality. Virtual Reality, 7:2-9.

30. Laurentini A (1994) The Visual Hull Concept for Silhouette-Based Image Understanding. IEEE Trans. Pattern Anal. Mach. Intell. 16, 2:150-162. 